\title{
Anti-de Sitter fragmentation
}

\section{Citation}

Maldacena, Juan, Jeremy Michelson, and Andrew Strominger. 1999. "Anti-de

Sitter Fragmentation." Journal of High Energy Physics 1999 (2): 011-011. https://

doi.org/10.1088/1126-6708/1999/02/011.

\section{Permanent link}

http://nrs.harvard.edu/urn-3:HUL.InstRepos:41417402

\section{Terms of Use}

This article was downloaded from Harvard University's DASH repository, and is made available under the terms and conditions applicable to Other Posted Material, as set forth at http:// nrs.harvard.edu/urn-3:HUL.InstRepos:dash.current.terms-of-use\#LAA

\section{Share Your Story}

The Harvard community has made this article openly available.

Please share how this access benefits you. Submit a story.

\section{Accessibility}


HUTP-98/A088

UCSBTH-98-8

hep-th/9812073

\title{
Anti-de Sitter Fragmentation
}

\author{
Juan Maldacena $^{1}$, Jeremy Michelson ${ }^{2,1}$ and Andrew Strominger ${ }^{1}$ \\ ${ }^{1}$ Department of Physics \\ Harvard University \\ Cambridge, MA 02138 \\ ${ }^{2}$ Department of Physics \\ University of California \\ Santa Barbara, CA 93106
}

\begin{abstract}
Low-energy, near-horizon scaling limits of black holes which lead to string theory on $A d S_{2} \times S^{2}$ are described. Unlike the higher-dimensional cases, in the simplest approach all finite-energy excitations of $A d S_{2} \times S^{2}$ are suppressed. Surviving zero-energy configurations are described. These can include tree-like structures in which the $A d S_{2} \times S^{2}$ throat branches as the horizon is approached, as well as disconnected $A d S_{2} \times S^{2}$ universes. In principle, the black hole entropy counts the quantum ground states on the moduli space of such configurations. In a nonsupersymmetric context $A d S_{D}$ for general $D$ can be unstable against instanton-mediated fragmentation into disconnected universes. Several examples are given.
\end{abstract}




\section{Introduction}

By now a beautiful and coherent story has been developed for the $A d S_{D} / C F T_{D-1}$ duality [1] for several values of $D$. A notable exception is the enigmatic case $D=2$. This case is perhaps the most interesting from the point of view of black hole physics because it is the very-near-horizon geometry of all known cases of supersymmetric black holes with non-zero entropy. 1 - One immediately puzzling feature is the fact that $A d S_{2}$ has two disconnected boundaries. From this alone it is evident that the $D=2$ case must involve qualitatively new features. Some preliminary progress on this case was reported in [3, 曲, 5,2,6] .

In this paper we shall continue exploration of the $A d S_{2} / C F T_{1}$ duality. In section 2 we analyze several inequivalent approaches to the near-horizon limit. We shall see that it is not possible to keep the charge, energy and temperature fixed in the usual manner while taking the Planck mass $M_{p} \rightarrow \infty$. In the most straightforward near-horizon limit the excitation energy of $A d S_{2}$ is forced to zero. The resulting theory describes only the (many) extremal black hole ground states.

$A d S_{2}$ is not the only zero-energy configuration which survives the $M_{p} \rightarrow \infty$ limit. In addition one can have geometries which are asymptotic to $A d S_{2}$ at large radius but branch (in a tree-like structure) into smaller $A d S_{2}$ regions as one moves toward the horizon. In section 3 we describe these configurations and their low energy dynamics. We further discuss their description in the dual CFT.

In section 4 we consider general $A d S_{D}$ spaces and discuss the possibility of brane creation by the antisymmetric D-form field strength [7,8]. If $D>2$, it can only occur in non-supersymmetric cases. We present an example of a non-supersymmetric $A d S_{3} \times S^{3} \times$ $K 3$ compactification where this seems to be the dominant decay mode. Finally we discuss topology changing instantons in the supersymmetric $A d S_{2}$ case. These instantons, found by Brill [9], describe tunneling between several $A d S_{2}$ spaces.

1 In some cases one encounters quotients of $A d S_{3}$, but these also reduce to $A d S_{2}$ at sufficiently low energies. [2] 


\section{2. $A d S_{2}$ as a Low-Energy Limit}

The oldest and simplest example of $A d S_{2}$ arising as a near-horizon geometry is in the context of the Reissner-Nordström solution of four-dimensional Einstein-Maxwell gravity. The full magnetically-charged solution is

$$
\begin{aligned}
d s^{2} & =-\frac{\left(r-r^{+}\right)\left(r-r^{-}\right)}{r^{2}} d t^{2}+\frac{r^{2}}{\left(r-r^{+}\right)\left(r-r^{-}\right)} d r^{2}+r^{2} d \Omega_{2}^{2} \\
F & =Q \epsilon_{2} \\
r_{ \pm} & =Q L_{p}+E L_{p}^{2} \pm \sqrt{2 Q E L_{p}^{3}+E^{2} L_{p}^{4}}
\end{aligned}
$$

In this expression $L_{p}$ is the Planck length $\left(L_{p}^{2}=G_{N}\right), \epsilon_{2}$ is the volume element on the unit $S^{2}$ and

$$
E=M-\frac{Q}{L_{p}}
$$

is the excitation energy above extremality. String theoretic examples generically involve more fields and several charges. We will mainly consider the Reissner-Nordström example because it is simpler and has qualitatively similar behavior.

An important feature of these black holes [10] is that the semiclassical analysis of their thermodynamic behavior breaks down very near extremality. This follows from the formulae for the entropy and Hawking temperature

$$
\begin{aligned}
S_{B H} & =\frac{\pi r_{+}^{2}}{L_{p}^{2}}, \\
T_{H} & =\frac{r_{+}-r_{-}}{4 \pi r_{+}^{2}} .
\end{aligned}
$$

Near extremality the energy-temperature relation is

$$
E \sim 2 \pi^{2} Q^{3} T_{H}^{2} L_{p}
$$

The energy of a typical quantum of Hawking emission is of order $T_{H}$. When this energy is of order of or greater than the total available energy $E$ above extremality, the semiclassical analysis must break down. This occurs at an excitation energy of order

$$
E_{g a p} \equiv \frac{1}{Q^{3} L_{p}}
$$


In string theory examples the nature of this breakdown is well-understood [11]: the black hole has a mass gap and (2.5) is the energy of its lowest-lying excitation2. In the description of a four-dimensional black hole given in [12] the gap state is the lowest excitation of a conformal field theory on a circle. In more general stringy constructions of four-dimensional black holes it is not always possible to compute the gap but the semiclassical analysis of [10] indicates it will always be of the order (2.5).

The near-horizon limit is simplest to describe for the extremal case in which $E=0$, $r_{+}=Q L_{p}$ and $T_{H}=0$. One then considers the limit

$$
L_{p} \rightarrow 0
$$

with

$$
U=\frac{r-r_{+}}{L_{p}^{2}}, \quad Q \text { fixed }
$$

The metric then reduces to

$$
\frac{d s^{2}}{L_{p}^{2}}=-\frac{U^{2}}{Q^{2}} d t^{2}+\frac{Q^{2}}{U^{2}} d U^{2}+Q^{2} d \Omega_{2}^{2}
$$

In null coordinates

$$
u^{ \pm}=\arctan \left(t \pm \frac{Q^{2}}{U}\right)
$$

the metric is

$$
\frac{d s^{2}}{L_{p}^{2}}=-\frac{4 Q^{2} d u^{+} d u^{-}}{\sin ^{2}\left(u^{+}-u^{-}\right)}+Q^{2} d \Omega_{2}^{2} .
$$

This is known as the Robinson-Bertotti geometry on $A d S_{2} \times S^{2}$. As illustrated in figure 1, the $A d S_{2} \times S^{2}$ region of the full Reissner-Nordström geometry is a ribbon which zigzags its way up through the infinite chain of universes. One of the timelike boundaries of $A d S_{2}$ $\left(u^{+}=u^{-}\right)$is just outside the black hole horizon, while the other $\left(u^{+}=u^{-}+\pi\right)$ is just inside.

2 There are however lower-energy modes describing the fragmentation of the black hole into smaller pieces. These modes are discussed below. 


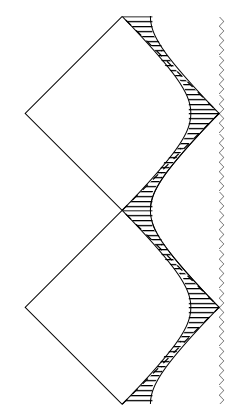

(a)

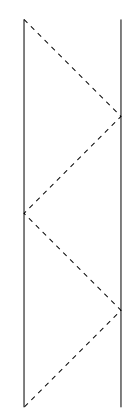

(b)

Fig. 1. (a) Penrose diagram corresponding to the extremal Reissner-Nordström black hole. The dashed line is the black hole horizon, and the shaded strip is the near-horizon $A d S_{2}$ region. (b) Penrose diagram for $A d S_{2}$. The diagonal lines are the horizons inherited from the embedding in Reissner-Nordström.

Since there are two timelike boundaries, quantum gravity with fermions on $A d S_{2}$ will have a NS and R sector. In the supersymmetric case, the Hamiltonian $H_{0}$ which generates time translations of the full solution (2.1) is the square of a supercharge: $H_{0}=Q^{2}$. This Hamiltonian generates accelerating trajectories in the global coordinates (2.10) of the nearhorizon $A d S_{2}$. The NS sector has a supercharge $\left(G_{1 / 2}+G_{-1 / 2}\right)$ which squares to $H_{0}$, but the $\mathrm{R}$ sector does not. Therefore in the supersymmetric case the near-horizon limit leads to the NS sector of quantum gravity on $A d S_{2}$.

One may also wish to consider more general limits which are not restricted to zero temperature and excitation energy. This problem is qualitatively different than its higherdimensional cousins because of the explicit factors of $L_{p}$ appearing in (2.4) and (2.5). In the following subsections we consider four such more general limits.

\subsection{Limit \# $1: L_{p} \rightarrow 0, \quad(E, Q)$ Fixed}

The limit $L_{p} \rightarrow 0$ with $(E, Q)$ fixed is problematic because according to (2.4) the Hawking temperature is infinite and the geometry is singular. Hence we do not know how to make sense of this limit. Note that for higher $(p>0) p$-branes with $A d S$ horizons the energy-temperature relation extracted from the near-extremal solutions is of the form

$$
E \sim V_{p} T_{H}^{p+1}
$$


This involves no explicit factors of $L_{p}$ (by dimensional analysis) and the $L_{p} \rightarrow 0$ limit is nonsingular for either fixed temperature or energy. In contrast the energy-temperature relation (2.5) is characteristic of a string-like rather than a point-like object. In place of the missing brane dimension a power of $L_{p}$ appears in (2.4).

\subsection{Limit \# $2: L_{p} \rightarrow 0,\left(T_{H}, Q\right)$ Fixed}

In order to keep the geometry of the solution fixed as $L_{p} \rightarrow 0$, one should keep the temperature $T_{H}$, which is related to the periodicity of the solution, fixed. From (2.4) it immediately follows that the excitation energy $E$ vanishes in such a limit, while the gap energy (2.5) goes to infinity. Defining $U$ exactly as in (2.7) one finds that the metric reduces to

$$
\frac{d s^{2}}{L_{p}^{2}}=-\frac{U\left(U+4 \pi Q^{2} T_{H}\right)}{Q^{2}} d t^{2}+\frac{Q^{2}}{U\left(U+4 \pi Q^{2} T_{H}\right)} d U^{2}+Q^{2} d \Omega_{2}^{2} .
$$

The metric (2.12) takes the canonical form (2.8) in the primed coordinates

$$
t^{\prime} \pm \frac{Q^{2}}{U^{\prime}}=\tanh \left[\pi T_{H}\left(t \pm \frac{1}{4 \pi T_{H}} \ln \frac{U}{U+4 \pi Q^{2} T_{H}}\right)\right]
$$

Hence at the classical level the geometry of the $L_{p} \rightarrow 0$ limit is independent of $T_{H}$.

At the quantum level there is a distinction. The quantum vacuum depends on the choice of time coordinate used to distinguish positive and negative frequency oscillators. The $S L(2, \mathbb{R})$ invariant vacuum leads to a thermal bath of particles in the coordinates (2.12). The energy density of this thermal bath for the case of a conformal matter system with central charge $c$ is

$$
T_{00}=\frac{c \pi T_{H}^{2}}{6}
$$

The impossibility of non-singular finite energy excitations of $A d S_{2} \times S^{2}$ at the classical level can be seen directly from the classical equations. The classical action in two dimensions contains the gravity-dilaton terms

$$
S_{2}=\frac{1}{4} \int d^{2} x \sqrt{-g}\left\{e^{-2 \phi}\left[R-F^{2}+2(\nabla \phi)^{2}\right]+\frac{2}{L_{p}^{2}}\right\}+S_{M} .
$$

where $S_{M}$ is the matter action, $R$ is the two dimensional scalar curvature, $4 \pi e^{-2 \phi}$ is the volume of the $S^{2}$ and $F=d A$ is the gauge field strength. The ++ constraint equation can be written

$$
-2 e^{-\phi} \nabla_{+} \nabla_{+} e^{-\phi}=T_{++} \geq 0
$$


Integrating (2.16) in conformal gauge $d s^{2}=e^{2 \rho} d u^{+} d u^{-}$with the measure $e^{\phi-2 \rho} d u^{+}$across $A d S_{2}$ from 0 to $\pi$ along the line $u^{-}=0$ gives

$$
\left.e^{-2 \rho} \partial_{+} e^{-\phi}\right|_{u^{+}=0}-\left.e^{-2 \rho} \partial_{+} e^{-\phi}\right|_{u^{+}=\pi}=\frac{1}{2} \int d u^{+} e^{\phi-2 \rho} T_{++} \geq 0 .
$$

$e^{-2 \rho}$ vanishes quadratically near the boundaries for $A d S_{2}$. If $T_{++}$is nonzero, then (2.17) implies that $e^{-\phi}$ must diverge linearly near at least one of the two boundaries. 3 Hence the geometry cannot be asymptotic to $A d S_{2} \times S^{2}$ when $T_{++}$is nonzero.

This zero-energy constraint might be modified at the quantum level in order to account for the energy of the Hawking radiation. Indeed the quantum constraints contain a oneloop correction from $c$ massless matter fields of the form $\frac{c}{12}\left(\partial_{+}^{2} \rho-\left(\partial_{+} \rho\right)^{2}\right)$ which modifies the preceding analysis.

The classical argument does not eliminate the possibility of black-hole-like spacetimes which have non-singular spacelike slices, but contain spacelike singularities in the past and future. For such spacetimes there may not be null surfaces which cross from one boundary to the other. A similar analysis using the time-time constraint equations on spacelike slices may yield relevant information, but one must consider the possibility of negative $T_{00}$ from tachyons.

Although the energy is classically constrained to vanish for nonsingular spacetimes, this limit is far from trivial because it should retain all the ground states of the system. The ground state entropy is $S=\pi Q^{2}$ for large $Q$. We have not succeeded in understanding the proper description of all these states in this framework. One possibility, discussed below, is that they arise from modes corresponding to black hole fragmentation or the separation of $A d S_{2} \times S^{2}$ universes.

\subsection{Limit \# $3: L_{p} \rightarrow 0,\left(E, T_{H}\right)$ Fixed, $Q \rightarrow \infty$}

In [3] it was found that Green functions in the near-horizon $A d S_{2} \times S^{2}$ geometry of the Reissner-Nordström black hole agreed with those of a $1+1$ chiral conformal field theory. The detailed agreement persisted when angular momentum was added to the black hole [3] and also for general charges [4]. Since Green functions measure correlations of finiteenergy disturbances these results suggest the existence of an $A d S_{2} / C F T_{1}$ correspondence involving non-zero excitation energies. This may seem to contradict the analysis of the

3 The rate of divergence depends on the coefficients in (2.15) which vary among different examples. 
previous subsection. However a somewhat different limit was implicit in [3]. One can hold both $E$ and $T_{H}$, as well as $E_{g a p}$, fixed as $L_{p} \rightarrow 0$ at the price of taking the charge $Q \sim L_{p}^{-1 / 3} \rightarrow \infty$. Since $Q$ is diverging this is a large $N$ limit. Defining

$$
V=\frac{r-r_{+}}{Q^{2} L_{p}^{2}}
$$

The metric (2.1) in the limit $L_{p} \rightarrow 0$ with fixed $T_{H}, E$ and $E_{\text {gap }}$ defined in (2.5) takes the $A d S_{2} \times S^{2}$ form

$$
\frac{\left(E_{g a p} L_{p}\right)^{2 / 3}}{L_{p}^{2}} d s^{2}=-V\left(V+4 \pi T_{H}\right) d t^{2}+\frac{1}{V\left(V+4 \pi T_{H}\right)} d V^{2}+d \Omega_{2}^{2} .
$$

The problem of small energies leading to divergent $\phi$ does not appear because $e^{-2 \phi}$, which appears on the left hand side of (2.16), is of order $Q^{2}$ and diverges for $L_{p} \rightarrow 0$. The right hand side is kept finite of order $E$ and can be neglected in comparison. The back reaction of matter on $\phi$ is suppressed. In string theory one also finds that the massive string modes decouple in this limit. Hence the limit largely consists of the free supergravity on $A d S_{2} \times S^{2}$. Nevertheless as seen in [3] an $A d S / C F T$ duality already has nontrivial content within this limit.

\subsection{Limit \# $4: L_{p}$ small, $\left(E, T_{H}, Q\right)$ Fixed}

Let us consider an infrared cutoff $U / Q^{2}<\Lambda$ on $A d S_{2} \times S^{2}$. In the dual CFT description this should correspond to an ultraviolet cutoff proportional to $\Lambda$. The cutoff theory should be capable of describing states with energies $E \ll \Lambda$. It follows from the discussion in section 2.2 that the addition of energy to $A d S_{2} \times S^{2}$ produces a dilaton which grows like $E U L_{p}^{2} / Q^{4}$ for large $U$ and small $E$. So if $E$ is small we can choose a cutoff which satisfies $E \ll \Lambda \ll Q / L_{p}$ so that the dilaton is small for all $U / Q^{2}<\Lambda$. Hence there should be a potentially useful approximate duality relating the cutoff theories at very low energies.

\section{3. $A d S_{2}$ Trees}

The $A d S_{2} \times S^{2}$ geometry (2.8) is not the unique classical charge $Q, E=0$ configuration which survives the $L_{p} \rightarrow 0$ limit with fixed $Q$. In addition there are classical solutions corresponding to BPS-saturated multi-black hole solutions with separations of order $L_{p}^{2}$ which survive as distinct objects in the limit $L_{p} \rightarrow 0$. These "tree" geometries are asymptotically $A d S_{2} \times S^{2}$ at large radius, but as one moves inward the geometry branches into smaller $A d S_{2} \times S^{2}$ regions. In a supersymmetric theory the quantum ground states are cohomology classes on the moduli space of such solutions. In principle one might understand the extremal black hole entropy by counting cohomology classes on this space. In this section we will discuss these configurations. 


\subsection{Two-Black Hole Configurations}

Let us begin with the asymptotically flat solution describing two Reissner-Nordström black holes (see fig. 2)

$$
\begin{aligned}
d s^{2} & =-V^{-2} d t^{2}+V^{2} d \vec{x}^{2}, \\
* F & =\frac{1}{L_{p}} d t \wedge d V^{-1}, \\
V & =1+\frac{Q_{1} L_{p}}{\left|\vec{x}-\vec{x}_{1}\right|}+\frac{Q_{2} L_{p}}{\left|\vec{x}-\vec{x}_{2}\right| .}
\end{aligned}
$$

Defining

$$
\begin{aligned}
\vec{U} & =\frac{\vec{x}}{L_{p}^{2}}, \\
\vec{U}_{1} & =\frac{\vec{x}_{1}}{L_{p}^{2}}, \\
\vec{U}_{2} & =\frac{\vec{x}_{2}}{L_{p}^{2}},
\end{aligned}
$$

and taking $L_{p} \rightarrow 0$, the near-horizon metric becomes

$$
\begin{aligned}
\frac{d s^{2}}{L_{p}^{2}} & =-V^{-2} d t^{2}+V^{2} d \vec{x}^{2}, \\
* F & =d t \wedge d V^{-1}, \\
V & =\frac{Q_{1}}{\left|\vec{U}-\vec{U}_{1}\right|}+\frac{Q_{2}}{\left|\vec{U}-\vec{U}_{2}\right|} .
\end{aligned}
$$

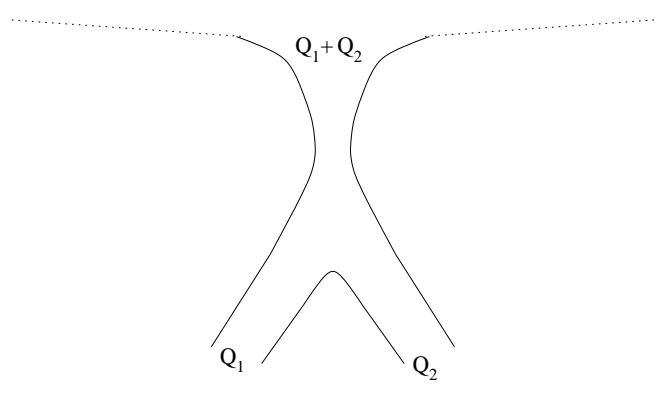

Fig. 2. A spatial cross-section the metric (3.1). There is an asymptotically Minkowskian region and a single charge $Q_{1}+Q_{2}$ throat region which divides into two throats of charges $Q_{1}$ and $Q_{2}$. In the $M_{p} \rightarrow \infty$ limit (3.3) the throat becomes infinitely long and the Minkowski region decouples. The splitting of the throat into two pieces survives this limit.

The difference $\vec{U}_{12}=\vec{U}_{1}-\vec{U}_{2}$ is a collective coordinate for the solution. The effective action for small variations of this coordinate is easily obtained by scaling the known 
result [13]

$$
S_{12}=\frac{1}{2}\left(Q_{1}^{3} Q_{2}+Q_{1} Q_{2}^{3}\right) \int d t \frac{\left(\partial_{t} \vec{U}_{12}\right)^{2}}{\left|\vec{U}_{12}\right|^{3}},
$$

and is finite for $L_{p} \rightarrow 0$. This geometry is locally flat with a conical singularity. It has the perhaps counterintuitive feature that the volume of the moduli space for widely separated black holes is very small, while that of nearly coincident black holes is divergent. The region corresponding to widely separated black holes is the point $\left|\vec{U}_{12}\right| \rightarrow \infty$ at the apex of the cone. Nearby black holes occupy the locally asymptotically flat region $\left|\vec{U}_{12}\right| \rightarrow 0$. It is possible that black hole entropy can be understood as the zero-energy states at the boundary of this moduli space.

Naively this divergent volume leads to an infinite number of arbitrarily low-lying excitations of near-coincident black holes. A similar divergence appears in counting the low-energy modes of a free scalar field in the vicinity of a black hole horizon because of arbitrarily large near-horizon redshifts. Presumably higher order corrections regulate the divergence in both cases, but we do not understand how this comes about.

\subsection{The Zerobrane Limit}

It is instructive to consider the case $Q_{1} \ll Q_{2}$. One can then view the charge $Q_{1}$ black hole as a charged BPS zerobrane in $A d S_{2} \times S^{2}$ with a constant electric field. The coupled action for such a zerobrane in $A d S_{2}$ is

$$
\begin{aligned}
S_{2}= & \frac{1}{4} \int d^{2} x \sqrt{-g}\left\{e^{-2 \phi}\left[R-F^{2}+2(\nabla \phi)^{2}\right]+\frac{2}{L_{p}^{2}}\right\} \\
& +\frac{1}{2} \oint d x \sqrt{h} e^{-2 \phi} K+\frac{Q_{1}}{L_{p}} \int A-\frac{Q_{1}}{L_{p}} \int d s .
\end{aligned}
$$

The scalar $\phi$ measures the size of the $S^{2}$ and the gauge field strength $F=d A$ is such that $e^{-2 \phi} F$ is of order $Q_{2}$. The metric on the boundary of $A d S_{2}$ is $h$ and $K$ is the extrinsic curvature of the boundary of $A d S$. The last two terms are the worldline action of the zerobrane.

Taking (3.3) and averaging $\vec{x}_{1}$ over the two sphere we get

$$
\begin{aligned}
\frac{d s^{2}}{L_{p}^{2}} & =-\frac{U^{2}}{Q_{2}^{2}} \frac{1}{h(U)^{2}} d t^{2}+\frac{Q_{2}^{2}}{U^{2}} h(U)^{2} d U^{2}, \\
e^{-\phi} & =Q_{2} h(U), \\
\frac{A}{L_{p}} & =\frac{U}{Q_{2}} \frac{1}{h(U)} d t,
\end{aligned}
$$


where

$$
h(U)=1+\frac{Q_{1}}{Q_{2}}\left(\Theta(U-a)+\frac{U}{a} \Theta(a-U)\right),
$$

where $\Theta(U)$ is the Heaviside step function. This represents a spherical distribution of zerobranes of total charge $Q_{1}$ hovering a fixed distance from the horizon. The zerobrane worldline is at $U=a$, where $a$ is the collective coordinate.

The solution (3.6) covers only the region outside the horizon. The analytic extension of this metric is provided by the Eddington-Finkelstein coordinatest

$$
d \bar{t}=d t-\left(1-\frac{Q_{2}^{2}}{U^{2}} h(U)^{2}\right) d U
$$

In these coordinates the solution is

$$
\begin{aligned}
\frac{d s^{2}}{L_{p}^{2}} & =-\frac{U^{2}}{Q_{2}^{2}} \frac{1}{h(U)^{2}} d \bar{t}^{2}+2\left(1-\frac{U^{2}}{Q_{2}^{2}} \frac{1}{h(U)^{2}}\right) d \bar{t} d U+\left(2-\frac{U^{2}}{Q_{2}^{2}} \frac{1}{h(U)^{2}}\right) d U^{2}, \\
e^{-\phi} & =Q_{2} h(U) \\
\frac{A}{L_{p}} & =\frac{U}{Q_{2}} \frac{1}{h(U)} d \bar{t} .
\end{aligned}
$$

Note that this is now regular at $U=0$, so we have extended the solution to $U<0$. However, at $U=-Q_{2} a / Q_{1}$, there is a singularity in the metric. This is an essential singularity of the solution; not only does the scalar curvature diverge at this point, but $e^{-\phi}$, which is the size of the internal $S^{2}$, is degenerating there (from (3.7)). This is an important contradistinction with pure $A d S_{2} \times S^{2}$.

Empty $A d S_{2} \times S^{2}$ has an inner and an outer boundary, both of which are nonsingular. We have just seen that (3.10) has the feature that the inner boundary is singular in our semiclassical description (see Figure 3). This suggests that the dual one-dimensional conformal field theory lives on the outer boundary alone, while some appropriate boundary conditions must be found to define the dynamics of the inner boundary.

4 The finite coordinate transformation is

$$
\bar{t}-\bar{t}_{0}=t-U-\frac{Q_{2}^{2}}{U} h(U)^{2}-2\left(\frac{Q_{1}^{2}}{a^{2}}(a-U)-\frac{Q_{1} Q_{2}}{a} \ln \frac{U}{a}\right) \Theta(a-U) .
$$




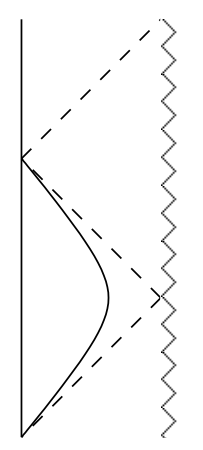

Fig. 3. The Penrose diagram for the solution (3.10). The curved path is the zero-brane worldline $U=a$, and the dashed line is the horizon $U=0$.

Another way to understand the appearance of a singularity in $(3.10)$ is to notice that for $U<a$ the harmonic function appearing in the metric (3.6) is of the form $h(U) / U \sim$ $1 / U+$ const so it is of the same form as that of the extremal Reissner-Nordström black hole before the near-horizon limit is taken.

\subsection{Charged Geodesics}

In the limit in which the back reaction of the zerobrane on the $A d S_{2} \times S^{2}$ geometry is neglected, it obeys a charged geodesic equation. This equation can be solved in general. We consider first the Euclidean case which is relevant for the discussion of instantons in section 4 .

It is easiest to calculate the zerobrane trajectories in Poincare coordinates

$$
d s^{2}=\frac{d t^{2}+d y^{2}}{y^{2}} .
$$

where the worldline action in 3.5 is

$$
S=m \int d t \frac{\sqrt{1+\left(\frac{d y}{d t}\right)^{2}}-1}{y} .
$$

The classical solutions to (3.12) are circles of arbitrary radius which are tangent to the boundary

$$
\left(t-t_{0}\right)^{2}+(y-a)^{2}=a^{2}
$$

as shown in fig. 4a. There is an additional solution

$$
y=y_{0}
$$


These solutions are transformed into one another by the action of $S L(2, \mathbb{R})$. In the strip coordinates

$$
d s^{2}=\frac{d \tau^{2}+d \sigma^{2}}{\sin ^{2} \sigma},
$$

the action becomes

$$
S=m \int d \tau \frac{\sqrt{1+\left(\frac{d \sigma}{d \tau}\right)^{2}}-\cos \sigma}{\sin \sigma}
$$

In this case the solutions are generically closed curves tangent to the boundary. For $t_{0}>0$, these are tangent to $\sigma=0$, and can be written

$$
\cosh \left(\tau-\tau_{0}\right)=\frac{\cos \left(\sigma_{m} / 2-\sigma\right)}{\cos \sigma_{m} / 2}
$$

as shown in fig. $4 \mathrm{~b}$. The $t_{0}<0$ solutions, tangent to $\sigma=\pi$, are mirror images of fig. $4 \mathrm{~b}$, including orientation. The additional solution is (see fig. 4c)

$$
e^{-\left(\tau-\tau_{0}\right)}=\sin \sigma .
$$

This trajectory has zero Euclidean energy and is relevant to vacuum tunneling. The timereverse of (3.18) is also a geodesic, corresponding to (3.13) with $t_{0}=0$.

Lorentzian trajectories can be obtained by Wick rotation. In Poincare coordinates they are (see fig. 4d)

$$
-\left(t-t_{0}\right)^{2}+(y+a)^{2}=a^{2}
$$

Similarly in the strip coordinates we get (see fig. 4e)

$$
\cos \left(\tau-\tau_{0}\right)=\frac{\sin \left(\sigma_{m} / 2-\sigma\right)}{\sin \left(\sigma_{m} / 2\right)}
$$

and its mirror image. Note that the particle gets to the boundary in finite global time. 

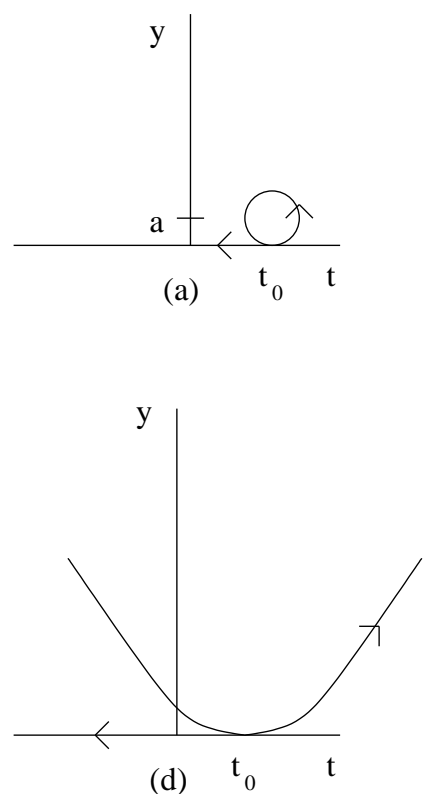

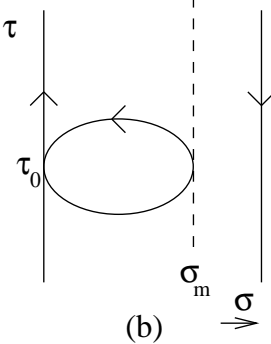

(b)

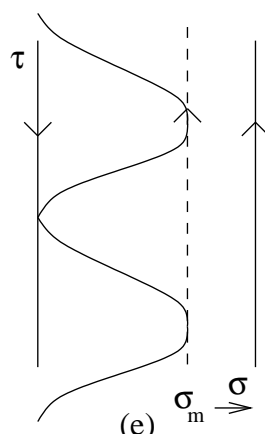

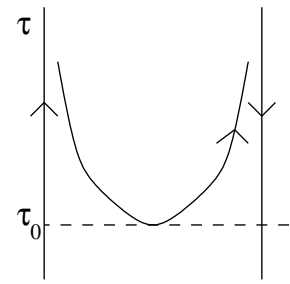

(c) $\stackrel{\sigma}{\rightarrow}$

Fig. 4. Trajectories of BPS charged particles in $A d S_{2}$ with Euclidean and Lorentzian signatures in various coordinate systems. The orientation of the arrows indicate the charges of the particles and the charges of the boundaries. (a) Euclidean trajectory in Poincare coordinates. (b) Euclidean trajectory in the strip coordinates. (c) Zero energy Euclidean trajectory in strip coordinates - this will be relevant for tunneling. (d) Lorentzian trajectory in Poincare coordinates. (e) Lorentzian trajectory in (global) strip coordinates.

\subsection{Multi-Black Hole Configurations}

The near-horizon two-black hole geometry (3.3) can be generalized to $n$ black holes simply by replacing $V$ in (3.3) with

$$
V=\sum_{i=1}^{n} \frac{Q_{i}}{\left|\vec{U}-\vec{U}_{i}\right|}
$$

The resulting geometry has $n A d S_{2} \times S^{2}$ regions near the $n$ horizons $\vec{x}=\vec{x}_{i}$ as well as an asymptotic one at large $\vec{x}$. Timelike singularities lie behind each of the $n$ horizons. The $3 n$-dimensional effective action deduced from [13] is

$$
S=\frac{3}{16 \pi} \int d t \sum_{i, j, k, l} Q_{i} Q_{j} Q_{k} Q_{l}\left|\partial_{t} \vec{U}_{i}-\partial_{t} \vec{U}_{j}\right|^{2} \int d^{3} U \frac{\left(\vec{U}-\vec{U}_{i}\right) \cdot\left(\vec{U}-\vec{U}_{j}\right)}{\left|\vec{U}-\vec{U}_{i}\right|^{3}\left|\vec{U}-\vec{U}_{j}\right|^{3}\left|\vec{U}-\vec{U}_{k}\right|\left|\vec{U}-\vec{U}_{l}\right|}
$$


Black holes encountered in string theory tend to involve more than just a single type of charge. This leads to non-trivial generalizations of (3.22). In five dimensions the black holes considered in [14] have three charges $Q_{1}, Q_{5}$ and $n$. The near-horizon geometry of a single black hole is $A d S_{2} \times S^{3}$. The moduli space geometry was studied in [15]. The near-horizon effective action for $p$ black holes is

$$
\begin{aligned}
S= & \frac{1}{4} \int d t \sum_{i \neq j}^{p} \sum_{k}^{p}\left(Q_{1 i} Q_{5 j} n_{k}+Q_{1 i} n_{j} Q_{5 k}+Q_{5 i} n_{j} Q_{1 k}\right)\left|\partial_{t} \vec{U}_{i}-\partial_{t} \vec{U}_{j}\right|^{2} \\
& \times\left[\frac{1}{\left|\vec{U}_{i}-\vec{U}_{j}\right|^{2}\left|\vec{U}_{i}-\vec{U}_{k}\right|^{2}}+\frac{1}{\left|\vec{U}_{i}-\vec{U}_{j}\right|^{2}\left|\vec{U}_{j}-\vec{U}_{k}\right|^{2}}-\frac{1}{\left|\vec{U}_{i}-\vec{U}_{k}\right|^{2}\left|\vec{U}_{j}-\vec{U}_{k}\right|^{2}}\right],
\end{aligned}
$$

where $U=r \sqrt{\frac{R V}{g^{2} \alpha^{\prime 4}}}$. In the sum, there are divergent terms when $k=i$ or $j$, but they cancel. Specializing to the case of two black holes, we have

$$
S=\frac{1}{2} \int d t \frac{\Gamma_{3}}{\left|\vec{U}_{1}-\vec{U}_{2}\right|^{4}}\left|\partial_{t} \vec{U}_{1}-\partial_{t} \vec{U}_{2}\right|^{2}
$$

where

$$
\Gamma_{3}=Q_{11} Q_{52} n_{2}+Q_{51} n_{2} Q_{12}+n_{1} Q_{12} Q_{52}+Q_{12} Q_{51} n_{1}+Q_{52} n_{1} Q_{11}+n_{2} Q_{11} Q_{51} .
$$

The four-dimensional multi-charge expression was found in [16]. Note that, like the singlecharge four dimensional case (3.4), the moduli space is locally flat. In fact, the two black hole case (3.24) is exactly $\mathbb{R}^{4} ; U=\infty$ is an ordinary point, and the 3 -sphere at $U=0$ is asymptotic infinity.

\subsection{The Dual CFT Picture}

Up to this point our discussion has been largely in the framework of semiclassical quantum gravity, and has not significantly involved string theory. In this section we discuss the $A d S_{2}$ trees in terms of the dual CFT on the boundary.

In the case of $A d S_{5}$ analogous multi-center gravity solutions (and D3 branes in $A d S$ ) correspond to Coulomb branches in the field theory where the scalar fields in the vector multiplets have expectation values and the gauge symmetry is broken to subgroups. Fields in a 0+1-dimensional quantum mechanical system do not have well defined expectation values; they fluctuate. The $A d S_{2}$ trees correspond to different classical vacua of a quantum mechanical theory and we expect that the system moves continuously among them. 
It seems natural to ask whether these trees correspond in any sense to the Coulomb or Higgs branches of the theory. In the classical $g \rightarrow 0$ limit of a D-brane system these two branches are very different. Quantum mechanically the system fluctuates and explores the whole moduli space. It has been argued in similar contexts in [17] [18] that the two branches decouple in the low energy limit and that the system, if initially in the Higgs branch, explores only the Higgs branch and does not wander on to the Coulomb branch. The tunneling processes discussed below seem to suggest that the branches are not decoupled. However this is not the end of the story, since it could be that branes in $A d S_{2}$ could correspond to something analogous to "small" instantons or similar configurations in the Higgs branch. For example in the $A d S_{5}$ description of Yang Mills field theory on $S^{4}$ a Yang Mills instanton — which corresponds to the Higgs branch of the $\mathrm{D}(-1)$ brane gauge theory — is a $\mathrm{D}(-1)$ brane moving in $A d S_{5}$ [19]. This implies that $\mathrm{D}(-1)$ branes in the near-horizon geometry are already dissolved in the D3-brane field theory and that we should interpret their positions as sizes. This analogy is imperfect because there is no Coulomb branch at all for the $S^{4}$ Yang-Mills theory. Nevertheless it suggests the possibility that a brane in $A d S_{2}$ or an $A d S_{2}$ tree could be corners of the Higgs branch near the point where the Coulomb branches meet. This view is corroborated by the observation that the volume of the moduli space for a 0-brane - as discussed above - in $A d S_{2}$ is finite near $U_{12}=\infty$.

\section{AdS Fragmentation}

In the previous section we discussed configurations in which a single charge $Q_{1}+Q_{2}$ $A d S_{2} \times S^{2}$ can branch into two $A d S_{2} \times S^{2}$ spaces with charges $Q_{1}$ and $Q_{2}$ as one moves spatially from infinity towards the horizon. As can be seen from the analysis of geodesics in section 3.3, this branching point can actually reach the boundary of $A d S_{2} \times S^{2}$ in finite global time. In principle the geometry could then fragment into two completely separate $A d S_{2} \times S^{2}$ universes with charges $Q_{1}$ and $Q_{2}$. Whether or not this actually happens depends on the boundary conditions at the $A d S_{2} \times S^{2}$ boundary.

In this section we will consider some examples in which fragmentation of a single $A d S$ universe into several smaller $A d S$ universe does occur, for both $A d S_{2}$ as well as higher dimensional examples. The processes we consider are tunneling processes mediated by topology-changing instantons. These or closely related instantons were considered in a different context in [7],8]. The first two subsections consider processes in which an initial $\operatorname{AdS}$

space fragments into one macroscopic and one microscopic component. The microscopic component is described by a brane. We then turn to the case (analyzed by Brill [9]) in which it splits into two macroscopic $A d S$ universes. 


\subsection{The Non-Supersymmetric Case}

Consider $A d S_{D}$ for general $D$ endowed with a constant antisymmetric $D$-form field strength. In flat space, a constant antisymmetric $D$-form field strength leads to $(D-2)$ brane creation for any variety of $(D-2)$-brane that is charged under the $D$-form field strength [8]. In the case of $D=2$ the field strength is a two-form and this reduces to the well known Schwinger pair production of 0-brane anti-0-brane pairs. The Schwinger process is described by an instanton in which the charged particle moves in a circular trajectory in the electromagnetic field. The vacuum decay rate is proportional to $e^{-S_{e}}$ where $S_{e}$ is the action of this Euclidean solution. The configuration to which the vacuum decays - namely a 0-brane anti-0-brane pair - is found by cutting the instanton in half at the moment of time symmetry (say $\tau=0$ ). The branes subsequently accelerate off to infinity. For general $D$ the analogous Euclidean solution is a $D-1$ sphere. Cutting it in half we get at $\tau=0$ a $D-2$ sphere. In the subsequent Lorentzian evolution the sphere expands due to the force exerted by the $D$-form field strength. In flat space this process of brane creation screens the $D$-form field strength. In $A d S_{D}(D-2)$-branes can also be created in this fashion [7].

We will here describe these intantons in the test-brane approximation, where we neglect the charge of the brane compared with the total flux of $F_{D}$ in $A d S_{D}$. Let us write the metric of $A d S_{D}$ as

$$
d s^{2}=R^{2}\left(\cosh ^{2} \rho d \tau^{2}+d \rho^{2}+\sinh ^{2} \rho d \Omega_{D-2}^{2}\right),
$$

where $R$ is the anti-de Sitter radius. Then the action of a spherically symmetric brane coupled to the $D$-form field strength is

$$
S=T R^{D-1} \Omega_{D-2} \int d \tau\left[\sinh ^{D-2} \rho \sqrt{\cosh ^{2} \rho+\left(\frac{d \rho}{d \tau}\right)^{2}}-q \sinh ^{D-1} \rho\right],
$$

where $T$ is the brane tension, $q$ is the ratio of the charge of the brane to its tension and

$$
\Omega_{D-2}=\frac{2 \pi^{\frac{(D-1)}{2}}}{\Gamma\left(\frac{D-1}{2}\right)}
$$

is the volume of a unit $D-2$ sphere. In the BPS case the forces balance so $q=1$. In a supersymmetric theory the BPS bound implies that $q \leq 1$ for all possible branes. In 
a non-supersymmetric context $q>1$ is possible (for example the electron). In the next subsection an example is given in string theory.

Now we turn to solutions of the brane action (4.2) for $q>1$. Since the action (4.2) is independent of time, Euclidean energy is conserved. For a spherically symmetric and compact surface, this energy is zero. Energy conservation then implies

$$
\frac{\cosh ^{2} \rho}{\sqrt{\cosh ^{2} \rho+\dot{\rho}^{2}}}-q \sinh \rho=0
$$

which is independent of $D$. The solution of this equation is

$$
\cosh \rho=\frac{\cosh \rho_{\max }}{\cosh \tau}
$$

where $\tanh \rho_{\max }=1 / q$. Equation (4.5) describes a closed $D-1$ surface with maximum radius $\rho_{\max }$. The action of this instanton is

$$
\begin{aligned}
S_{\text {inst }} & =\frac{2 T R^{D-1} \Omega_{D-2}}{\sinh \rho_{\max }} \int_{0}^{\rho_{\max }} d \rho \frac{\sinh ^{D-2} \rho \sqrt{\sinh ^{2} \rho_{\max }-\sinh ^{2} \rho}}{\cosh \rho} \\
& =\frac{\pi^{\frac{D}{2}} T R^{D-1}}{\Gamma\left(\frac{D+2}{2}\right)} \sinh ^{D-1} \rho_{\max } F\left(1, \frac{D-1}{2} ; \frac{D+2}{2} ;-\sinh ^{2} \rho_{\max }\right) .
\end{aligned}
$$

At $\tau=0$ one can match (4.5) to the Lorentzian solution

$$
\cosh \rho=\frac{\cosh \rho_{\max }}{\cos \tau}
$$

which describes the post-tunneling evolution. Note that the brane gets to the boundary $($ at $\rho=\infty$ ) in finite time (at $\tau=\pi / 2$ ) (see fig. 5).

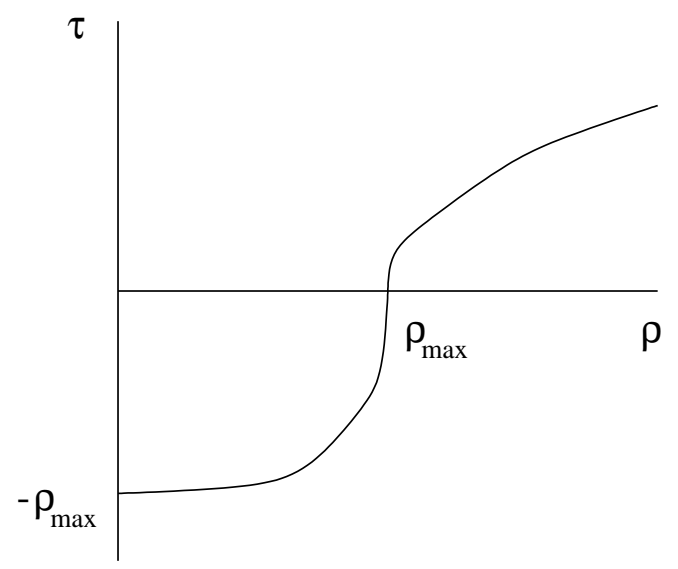

Fig. 5. The Euclidean instanton (4.5) for $\tau<0$ matches onto the Lorentzian solution (4.7) at $\tau=0$. 
If $q<1$ the Euclidean solution is

$$
\cosh \rho=\frac{\sinh \tau_{\max }}{\sinh \tau} \quad \tanh \tau_{\max } \equiv q
$$

It does not describe a tunneling process because the solution doesn't have a moment of time symmetry and also its action is infinite. This is expected since tunneling is forbidden by energy conservation for $q<1$.

\subsection{An Example of a Brane With Charge Greater Than Tension}

In this section we will give an example of a non-supersymmetric $A d S_{3} \times S^{3} \times K 3$ compactification which is unstable to fragmentation. Consider type IIB on $K 3$. The six dimensional theory has an $S O(5,21)$ multiplet of strings, coming from branes wrapped on various cycles of $K 3$. A string is characterized by a charge vector $q^{I}$ transforming in the vector under $S O(5,21)$. If $q^{2}>0$ the string is BPS and the near-horizon geometry is $A d S_{3} \times S^{3} \times K 3$. If $q^{2}<0$ the string will not be BPS, but nevertheless there is a supergravity solution and its near-horizon geometry is again $A d S_{3} \times S^{3} \times K 3$ [20]. A simple example is the following. Take a set of $Q_{5} \mathrm{D} 5$-branes wrapped on $K 3$ which leads to a string in six dimensions. We can add D1-branes to this system. Supersymmetry is preserved only if the D1-branes have the right charge - we choose it to be positive. The BPS bound for the tension of the string is $T \sim\left|Q_{5} V_{4}+Q_{1}\right| / g$ where $V_{4}$ is the volume of $K 3$ in string units. When we wrap a D5-brane on $K 3$ there is one unit of negative D1-brane charge induced on the brane [21]. So there are BPS strings with charges $\left(q_{5}, q_{1}\right)=(1,-1)$ whose tension is $T_{(1,-1)}=\left(V_{4}-1\right) / g$ (we are assuming $V_{4}>1$ ). Supersymmetric black strings will have charges $Q_{1} \geq 0$. Consider a black string whose charge is $Q_{1}<0$. The bosonic part of the supergravity equations have a structure which is not very sensitive to the relative sign of $Q_{1}$. Actually the only change in the solution is the sign in the electric part of the $B$ field. In particular, the volume of the $K 3$ at the horizon, (encoded in the dilaton), will be $V=\left|Q_{1}\right| / Q_{5}$ so we will take $\left|Q_{1}\right|>Q_{5}$ so that the above formula for the tension of the $(1,-1)$ string is correct.

We can also study small fluctuations around this solutions and check that there is no tachyon producing an instability (i.e. all tachyons obey the Breitenlohner-Freedman bound $\left.m^{2} \geq-1\right)$. The basic reason is that the equations involve a coset space $S O(5,21) / S O(5) \times$ $S O(21)$ and self-dual field strengths transforming in the $\mathbf{5}$ of $S O(5)$ and antiself-dual field strengths transforming in the $\mathbf{2 1}$. In the case that $Q_{1}>0$ only the self-dual field strength 
is non-zero while if $Q_{1}<0$ only the antiself-dual part is non-zero in the near horizon region. The bosonic equations are symmetric under the exchange of self-dual and antiselfdual fields together with the change of the $S O(5)$ and $S O(21)$ pieces of the coset. So if for $Q_{1}>0$ we had 21 fixed scalars and a similar number of "good" tachyons now we will have 5 fixed scalars and a similar number of "good" tachyons, etc. In the negative $Q_{1}$ case the string with charges $\left(q_{5}, q_{1}\right)=(1,-1)$ will feel a repulsive force, since the only difference with the supersymmetric case (in which the forces balance) is that the onebrane electric force is repulsive rather than attractive. So this string will have $q>1$ in the notation of the previous section (more precisely $q=\frac{\left|Q_{1}\right|+Q_{5}}{\left|Q_{1}\right|-Q_{5}}$ ). This implies that the black hole would lose its charge by emitting these $q>1$ branes.

It is natural to ask whether other non-supersymmetric $A d S$ spaces would have similar instabilities. These kind of instabilities would generically occur for $A d S_{2}$ cases; a well known example is an extremal electrically charged black hole in our universe, which discharges by emitting electrons (which have $q \sim 2 \times 10^{21}$ ). It can be seen that if we have an $A d S_{5} \times M^{5}$ compactification where $M^{5}$ is an Einstein manifold, then the gravity equation of motion implies that a three-brane moving in that geometry will have $q=1$ regardless of whether supersymmetry is broken or not. There could be, however, other branes with $q>1$ if the Einstein manifold has some small cycles on which one can wrap branes etc. In cases where one has a "warped" geometry [22], i.e. a solution where the radius of $A d S_{5}$ depends on the coordinates on $M^{5}$ then the threebrane could have $q>1$. An example is the compactification that has $S O(5)$ symmetry that arises as an unstable IR "fixed point" after perturbing the $\mathcal{N}=4$ theory by a relevant operator [23].

The nature of the tunneling process is illuminated by consideration of the energy of a (momentarily) static spherical brane at radius $\rho$. This is not an equilibrium configuration. This energy has a positive contribution from the mass of the brane and a negative contribution from the electric potential:

$$
E(\rho)=T R^{D-2} \Omega_{D-2}\left[\sinh ^{D-2} \rho \cosh \rho-q \sinh ^{D-1} \rho\right]
$$

We see that if $q>1$ then $E(\rho) \rightarrow-\infty$ as $\rho \rightarrow \infty$. So the system decays in order to reach this lower energy configuration. If $q=1$ then we see that $E(\rho) \rightarrow\{\infty$, const, 0$\}$ for the $\{D>3, D=3, D=2\}$ cases as $\rho \rightarrow \infty$. This is consistent with the fact that only for $D=2$ can we have tunneling in the $q=1$ case.

It is interesting to note that in the $A d S_{3}$ case the constant value of the energy is related to the change in the central charge of the system when we remove a brane. This is 
anticipated from the dual NS-sector CFT description in which the ground state energy is proportional to the central charge. More precisely, if we have a D5-D1 brane system then the change in energy is $Q_{1} / 2$ if we remove a fivebrane and $Q_{5} / 2$ if we remove a onebrane. This further implies that if we have enough energy above the ground state in $A d S_{3}$ then the system can decay by emitting branes. In the case with NS charges (NS fivebranes and fundamental strings) this decay mode might be related to the negative norm states for the $S L(2, \mathbb{R})_{Q_{5}}$ WZW model arising when the square of the mass of the state is of the order of $Q_{5}$.

\subsection{The Supersymmetric Case}

In this subsection we consider the supersymmetric case $q=1$. The solution to the geodesic equation for $q=1$ is

$$
e^{\tau}=\cosh \rho
$$

as discussed previously in (3.18). Unlike the $q<1$ case (4.10) is not a "bounce" solution. It does not have a moment of time symmetry and there is no negative mode indicating an instability. Rather it represents tunneling between two degenerate vacua. At $\tau=-\infty$ the instanton is asymptotic to charge $\left(Q_{1}+Q_{2}\right) A d S_{2}$, while at $\tau=+\infty$ one has charge $Q_{2}$ $A d S_{2}$ plus a charge $Q_{1}$ brane in the boundary.

For $D>2$ the instanton action is infinite so the tunneling does not actually occur. For $D=2$ it takes the finite value

$$
S_{\text {instanton }}=\pi Q_{1} Q_{2}
$$

Comparing with (2.3) we see that this can be written

$$
S_{\text {instanton }}=-\frac{1}{2} \Delta S_{B H}
$$

where $\Delta S_{B H}$ is the difference in the Bekenstein-Hawking entropy of the initial and final states. In a description which microscopically accounts for the Bekenstein-Hawking entropy, twice the factor (4.12) would arise in transition probabilities from averaging over initial and summing over final states. This agrees with the fact that the instanton (4.12) gives transition amplitudes. Apparently the instanton mysteriously knows the number of microstates. Previous examples of instantons counting microstates in this fashion can be found in [24], [9]. 
All supersymmetric instantons will have fermion zero modes which we have not analyzed in detail. This means that the transitions will be accompanied by a change in fermion number and/or spacetime momentum, 目 and will not shift the ground state energy.

\subsection{The Brill Instanton}

A tunneling process in which an initial $A d S_{2} \times S^{2}$ universe fragments into two final $A d S_{2} \times S^{2}$ universes should be described by a smooth instanton with one initial and two final $A d S_{2} \times S^{2}$ boundaries. Such an instanton was discovered by Brill [9], whose work we review in this subsection. In the limit in which one of the final universes is small and can be treated like a brane, this instanton reduces to (4.10) of the previous subsection.

The Euclidean action for the Einstein-Maxwell theory is

$$
S=-\frac{1}{16 \pi} \int d^{4} x \sqrt{g}\left(R-F^{2}\right)-\frac{1}{8 \pi} \oint d^{3} x \sqrt{h} K,
$$

where $K$ and $h$ are the trace of the extrinsic curvature and the induced metric on the boundary and we have set Newton's constant to one. This has the family of solutions

$$
\begin{aligned}
d s^{2} & =V^{2} d \vec{x}^{2}+V^{-2} d w^{2}, \\
* F & =-d w \wedge d V^{-1}, \\
\vec{\nabla}^{2} V(\vec{x}) & =0
\end{aligned}
$$

where $\vec{\nabla}^{2}$ is the Laplacian on flat $\mathbb{R}^{3}$. The special case

$$
V=\frac{Q_{0}}{|\vec{x}|}
$$

corresponds to the Euclidean $A d S_{2} \times S^{2}$ Robinson-Bertotti universe with magnetic charge $Q_{0}$ on the $S^{2}$, and $A d S_{2}$ cosmological constant $\frac{2}{Q_{0}}$. The Brill instanton is 6

$$
V=\frac{Q_{1}}{\left|\vec{x}-\vec{x}_{1}\right|}+\frac{Q_{2}}{\left|\vec{x}-\vec{x}_{2}\right|}
$$

For $\vec{x} \rightarrow\left(\vec{x}_{1}, \vec{x}_{2}, \infty\right)$ the metric given by (4.16) approaches the $A d S_{2} \times S^{2}$ metric (4.15) with charge $Q=\left(Q_{1}, Q_{2}, Q_{0}=Q_{1}+Q_{2}\right)$.

5 This momentum refers to the original asymptotically flat region. In the $A d S$ context it is conjugate to zero modes of singleton fields.

6 This metric is obtained by analytic continuation $t \rightarrow i w$ of (3.3), but we will not interpret $w$ as Euclidean time. 
We wish to interpret this as a semiclassical contribution to the tunneling of an initial charge $Q_{0}=Q_{1}+Q_{2} A d S_{2} \times S^{2}$ spacetime to final charge $Q_{1}$ and $Q_{2}$ spacetimes. In order to do so we need to identify one initial surface $\Sigma_{0}$ and two final surfaces $\Sigma_{1}$ and $\Sigma_{2}$ with topologies $\mathbb{R} \times S^{2}$ corresponding to spatial slices of $A d S_{2} \times S^{2}$ spacetimes with the appropriate charges. The metrics on the surfaces should agree with those of the corresponding slices of $A d S_{2} \times S^{2}$. The extrinsic curvatures should vanish so that the continuation back to Lorentzian signature gives real initial data. 7

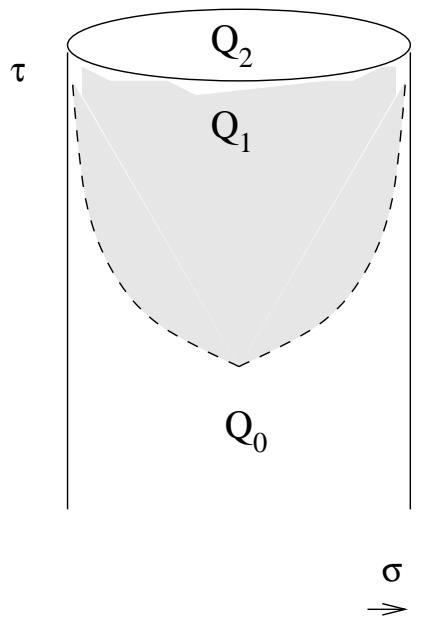

Fig. 6. The Brill instanton. We start with an charge $Q_{1}+Q_{2} A d S_{2} \times S^{2}$ space which splits into two $A d S_{2} \times S^{2}$ spaces of charges $Q_{1}$ and $Q_{2}$. For finite Euclidean time they still look like a single charge $A d S$ space close to the boundary. Only at infinite time do the two $A d S$ spaces separate.

Let us introduce the following variable

$$
y=\left(\frac{Q_{1}}{\sqrt{\left|\vec{x}-\vec{x}_{1}\right|}}+\frac{Q_{2}}{\sqrt{\left|\vec{x}-\vec{x}_{2}\right|}}\right)^{2}
$$

And then define the variables $\tau, \sigma$ through

$$
w+i y=e^{\tau+i \sigma}
$$

Where $0 \leq \sigma \leq \pi$. We see that for $\tau \rightarrow-\infty$ we have the single charge $Q_{0} A d S$ space in the (Euclidean) strip coordinates and for large $\tau$ we have two $A d S_{2}$ spaces of charges $Q_{1}$ and

7 The surfaces given in [9] differ from those described here and do not satisfy this criterion. 
$Q_{2}$ which actually meet at $\sigma \rightarrow 0, \pi$ to form again a charge $Q_{0} A d S_{2}$ space (the change of variables (4.17) is, in a sense, double valued). The point $\sigma$ where they meet goes to the boundary $(\sigma=0, \pi)$ when $\tau \rightarrow+\infty$ so that in the limit we really have two disconnected $A d S_{2}$ spaces. We need to regulate the spatial extent of $A d S_{2}$ (the $\sigma$ coordinate) and also the temporal extent. This could be achieved by taking cutoffs $\epsilon<\sigma<\pi-\epsilon$ and $-T<\tau<T$. Notice that if we take first a finite $\epsilon$, then the two final $A d S_{2}$ regions become disconnected at finite $T$.

The tunneling amplitude is proportional to the exponential of minus the instanton action. Reducing the action to a surface term and subtracting the action of the vacuum to vacuum instanton, Brill finds an amplitude proportional

$$
A_{Q_{0} \rightarrow Q_{1}+Q_{2}} \sim e^{\frac{1}{2} \Delta S_{B H}}
$$

where

$$
\frac{1}{2} \Delta S_{B H}=\frac{1}{2}\left\{S_{B H}\left(Q_{1}\right)+S_{B H}\left(Q_{2}\right)-S_{B H}\left(Q_{1}+Q_{2}\right)\right\}=-\pi Q_{1} Q_{2} .
$$

In this expression

$$
S_{B H}(Q)=\pi Q^{2}
$$

is the Bekenstein-Hawking entropy for a charge $Q$ extremal black hole. Squaring the amplitude to get the transition probability one finds that it is proportional to minus the exponential of the entropy decrease, as expected. This result agrees exactly with the result (4.11) computed for $Q_{1} \ll Q_{2}$. Because of the necessity of subtractions, it is not manifestly obvious (although it is expected) that the action will be the same when computed for the initial and final surfaces described above.

An interpretation of the tunneling process which does not refer to the decoupled asymptotically flat region can be given in the context of third-quantized Hilbert space in which states are labeled by the occupation numbers $n_{i}$ of $A d S_{2} \times S^{2}$ spacetimes with charges $Q_{i}$. The Brill instanton corresponds to a nonperturbative correction to the Hamiltonian which changes these occupation numbers. Due to charge conservation there are superselection sectors labeled by the total charge. In the semiclassical approximation considered here, the $A d S_{2} \times S^{2}$ spacetimes are like non-relativistic particles. There is no pair-creation of oppositely charged spacetimes, and $Q_{i}$ is restricted to be positive.

In general topology-changing processes which change the number of universes are problematic (a review can be found in [25]). Among other reasons, it is difficult to describe such processes by a Hamiltonian because there is in general no canonical way to compare 
the times of different universes. This problem cannot arise in the present context because it was derived as a limit of a system which included the asymptotically flat region and did have a Hamiltonian. The separate $A d S_{2} \times S^{2}$ universes carry a preferred time with them as a remnant of the asymptotically flat region which once joined them.

\section{Acknowledgements}

We have benefitted from useful conversations with R. Britto-Pacumio, J. de Boer, M. Headrick, K. Hori, H. Ooguri, E. Silverstein, M. Spradlin, J. Stopple, L. Susskind and E. Witten. This work was supported in part by an NSF Graduate Fellowship, an NSERC PGS B Scholarship and DOE grant DE-FGO2-91ER40654. 


\section{References}

[1] J. Maldacena, The Large N Limit of Superconformal Field Theories and Supergravity, Adv. Theor. Math. Phys. 2 (1998) 231-252, hep-th/9711200.

[2] A. Strominger, AdS $S_{2}$ Quantum Gravity and String Theory, hep-th/9809027.

[3] J. Maldacena and A. Strominger, Universal Low-Energy Dynamics for Rotating Black Holes, Phys. Rev. D 56 (1997) 4975-4983, hep-th/9702015.

[4] M. Cvetič and F. Larsen, Microstates of Four Dimensional Black Holes from NearHorizon Geometry, UPR-798-T, ITP/NSF-98-065, hep-th/9805146;

M. Cvetič and F. Larsen, Greybody Factors for Black Holes in Four Dimensions, Phys. Rev. D 57 (1998) 6297-6310, hep-th/9712118;

M. Cvetič and F. Larsen, Greybody Factors for Rotating Black Holes in Four Dimensions, Nucl. Phys. B506 (1997) 107-120, hep-th/9706071.

[5] H. J. Boonstra, B. Peeters and K. Skenderis, Brane intersections, Anti-de Sitter Spacetimes and Dual Superconformal Field Theories, Nucl. Phys. B533 (1998) 127-162, hep-th/9803231.

[6] Y. Satoh, BTZ Black Holes and the Near-Horizon Geometry of Higher-Dimensional Black Holes, PUPT-1816, hep-th/9810135.

[7] J. Brown and C. Teitelboim, Neutralization of the Cosmological Constant by Membrane Creation, Nucl. Phys. B297 (1988) 787-836.

[8] F. Dowker, J. Gauntlett, G. Gibbons and G. Horowitz, Nucleation of p-branes and Fundamental Strings, Phys. Rev. D 53 (1996) 7115-7128, hep-th/9512154.

[9] D. Brill, Splitting of an Extremal Reissner-Nordström Throat via Quantum Tunneling, Phys. Rev. D46 (1992) 1560-1565.

[10] J. Preskill, P. Schwarz, A. Shapere, S. Trivedi and F. Wilczek, Limitations on the Statistical Description of Black Holes, Mod. Phys. Lett. A6 (1991) 2353-2362.

[11] J. Maldacena and L. Susskind, D-branes and Fat Black Holes, Nucl. Phys. B475 (1996) 679-690, hep-th/9604042.

[12] J. Maldacena and A. Strominger, Statistical Entropy of Four-Dimensional Black Holes, Phys. Rev. Lett. 77 (1996) 428-429, hep-th/9603060.

[13] R. Ferrell and D. Eardley, Slow-Motion Scattering and Coalescence of Maximally Charged Black Holes, Phys. Rev. Lett. 59 (1987) 1617-1620.

[14] A. Strominger and C. Vafa, Microscopic Origin of the Bekenstein-Hawking Entropy, Phys. Lett. B379 (1996) 99-104, hep-th/9601029.

[15] D. M. Kaplan and J. Michelson, Scattering of Several Multiply Charged Extremal $D=5$ Black Holes, Phys. Lett. B410 (1997) 125-130; hep-th/9707021.

[16] J. Michelson, Scattering of Four-Dimensional Black Holes, Phys. Rev. D 57 (1998) 1092-1097; hep-th/9708091. 
[17] O. Aharony, M. Berkooz, S. Kachru, N. Seiberg and E. Silverstein, Matrix Description of Interacting Theories in Six Dimensions, Adv. Theor. Math. Phys. 1 (1998) 148-157, hep-th/9707079.

[18] E. Witten, On the Conformal Field Theory of the Higgs Branch, JHEP 07 (1997) 003, hep-th/9707093.

[19] T. Banks and M. Green, Nonperturbative Effects in $A d S^{5} \times S^{5}$ String Theory and $d=4$ SUSY Yang-Mills, JHEP 05 (1998) 002, hep-th/9804170;

C. S. Chu, P. M. Ho and Y. Y. Wu, D-Instanton in AdS $S_{5}$ and Instanton in $S Y M_{4}$, SISSA-58/98/FM, SHU-TIPAC-98007, hep-th/9806103;

I. Kogan, G. Luzon, D-Instantons on the Boundary, OUTP-98-49P, hep-th/9806197;

V. Balasubramanian, P. Kraus, A. Lawrence and S. Trivedi, Holographic Probes of Anti-de Sitter Spacetimes, HUTP-98/A057, CALT68-2189, Fermilab-Pub-98/240-T, hep-th/9808017;

M. Bianchi, M. Green, S. Kovacs and G. Rossi, Instantons in Supersymmetric Yang-Mills and D-Instantons in IIB Superstring Theory, JHEP 08 (1998) 013, hepth/9807033.

[20] M. J. Duff, H. Lü and C. Pope, $A d S_{3} \times S^{3}$ (Un)twisted and Squashed, and an $O(2,2 ; \mathbb{Z})$ Multiplet of Dyonic Strings, CTP TAMU-28/98, LPTENS-98/30, SISSA Ref. 79/98/EP, hep-th/9807173.

[21] M. Bershadsky, V. Sadov and C. Vafa, D-branes and Topological Field Theories, Nucl. Phys. B463 (1996) 420-434, hep-th/9511222.

[22] P. Van Nieuwenhuizen and N. Warner, New Compactifications of Ten Dimensional and Eleven Dimensional Supergravity on Manifolds Which Are Not Direct Products, Comm. Math. Phys. 99 (1985) 141;

C. Hull and N. Warner, Noncompact Gaugings From Higher Dimensions, Class. Quant. Grav. 5 (1988) 1517.

[23] M. Günaydin, L. Romans and N. Warner, Compact and Noncompact Gauged Supergravity Theories in Five Dimensions, Nucl. Phys. B272 (1986) 598;

L. Girardello, M. Terini, M. Porrati and A. Zaffaroni, Novel Local CFT and Exact Results on Perturbations of $N=4$ Super Yang Mills From AdS Dynamics, CERN-TH/98-323, IFUM-633-FT, IMPERIAL/TP/98-99/4, NYU-TH/98/10/03, hep-th/9810126;

J. Distler and F. Zamora, Non-supersymmetric Conformal Field Theories From Stable Anti-de Sitter Spaces, UTTG-14-98, hep-th/9810206.

[24] D. Garfinkle, S. B. Giddings and A. Strominger, Entropy in Black Hole Pair Production, Phys. Rev. D 49 (1994) 958-965; gr-qc/9306023.

[25] A. Strominger, Baby Universes, in TASI '88: Particles, Strings and Supernovae (ed. A. Jevicki and C.-I. Tan) World Scientific, 1989. 